\title{
Bone metabolism in spinal cord injured individuals and in others who have prolonged immobilisation. A review
}

\author{
D Uebelhart ${ }^{1,2}$, B Demiaux-Domenech ${ }^{3}, \mathrm{M} \mathrm{Roth}^{1}, \mathrm{~A}_{\text {Chantraine }}{ }^{1}$ \\ ${ }^{1}$ Division of Physical Medicine and Rehabilitation, University Hospital, Geneva, Switzerland; ${ }^{2}$ Department of \\ Biochemistry, Rush Presbyterian St Luke's Medical Center, Chicago, Illinois, USA; ${ }^{3}$ Division of \\ Pathophysiology, University Hospital, Geneva, Switzerland
}

Immobilisation or disuse is a condition known to be associated with a decrease in bone mass, osteopenia and in some people leading to osteoporosis with an increased risk of fractures. In this condition, previous histomorphometric and biochemical reports have shown an uncoupling between bone formation and resorption, but the exact sequence of the events resulting in bone loss is still not fully understood. In spinal cord injury for instance, the main finding soon after the onset is decreased osteoblastic activity associated with a dramatic increase in bone degradation. The overall consequence of these metabolic events is the development of a rapid and severe osteoporosis only observed in the paralysed part of the body associated with the loss of biomechanical strength and the biosynthesis of a structurally modified matrix which is unable to sustain normal mechanical stress. This situation dramatically increases the risk of fractures. The same uncoupling phenomenon has been described in healthy individuals who have been submitted to long duration bedrest and also in astronauts during spaceflight; but the timing, intensity and the metabolic subset may be different as these people do recover after cessation of the disuse period, which does not occur in paralysed patients. As new accurate and sensitive non-invasive techniques have become available recently to assess bone and connective tissue metabolism, more information is now available regarding bone loss in paralysed and/or immobilised individuals. These techniques should be definitely helpful in orientating new therapeutic trials with drugs and/or procedures intended to correct the musculoskeletal deleterious effects of disuse. This paper is therefore aimed at a review of bone metabolism in those with a severe spinal cord injury, or with a long duration of bedrest, or with loss of biomechanical function, or with actual or simulated spaceflight, in all instances using non-invasive techniques.

Keywords: bone metabolism; biochemical markers; densitometry; disuse osteoporosis; spinal cord injury; weightlessness; long-term bedrest; long term immobilisation

\section{Introduction}

Generic terms such as 'disuse' or 'immobilisation osteoporosis' have been used currently to describe the skeletal changes that occur in situations such as long-term bed rest, spaceflight or simulated weightlessness, loss of normal biomechanical function, and in neurological disorders associated with acute or chronic paralysis. The terminology used certainly does not reflect the aetiopathogenic factors involved in the occurrence of bone loss in any of these situations which are most likely to appear multifactorial in most of the examples. Nevertheless the loss of the normal biomechanical stress associated with some degree of

Correspondence: D Uebelhart, Department of Clinical Neurosciences \& Dermatology, Division of Physical Medicine \& Rehabilitation, University Hospital - Beau Sejour, 1211 Geneve 14/ Switzerland

This paper is dedicated to the memory of Pierre Minaire neurovascular changes due to modification of autonomic nervous system activity have metabolic consequences where bone tissue is one of the primary targets. This paper is aimed at making a short review of the major changes of bone metabolism in those with paralysis as seen in spinal cord injury patients, as compared to what is observed in healthy subjects submitted to a long duration of bedrest, or of microgravity. The authors concentrated on the results of recent non-invasive techniques such as bone densitometry and biochemical markers of bone and connective tissue metabolism to assess the effects of disuse.

\section{Bone metabolism in spinal cord injury patients}

In spinal cord injury patients (SCI), some alterations in bone metabolism can be reproducibly observed including a rapid and intense bone loss occurring predominantly in the paralysed limbs, hypercalcemia and 
hypercalciuria, and increased hydroxyproline excretion. An invasive technique such as bone histomorphometry has demonstrated a $33 \%$ decrease of the trabecular bone volume measured on iliac crest biopsies associated with increased bone resorption surfaces, a reduced bone formation rate ${ }^{1}$ and an increased bone marrow fat content. ${ }^{2}$ Using non-invasive techniques, such as dual-photon absorptiometry (DPA) it is possible to quantify bone loss more accurately. Using DPA, many studies have confirmed the rapid and important decrease of bone mass. Biering-Sorensen et al have observed in a 3 years prospective study a $50 \%$ decrease in bone mineral content in the paralysed limbs of SCI patients, mostly at the femoral neck and the proximal metaphysis of the tibia. ${ }^{3}$ Bone loss in the paralysed segments of the body has also been studied by transversal studies. ${ }^{4-7}$ More recently it has been demonstrated that total-body bone mass was also significantly decreased by $\sim 12 \%$ in paraplegic subjects ${ }^{6}$ and indeed all skeletal sites appeared to be concerned, with the exception of the skull. ${ }^{8}$ Recent data originating from our group confirm that bone mass is directly affected by paralysis, but muscle mass and fat mass are also affected. Using dual X-ray absorptiometry to assess whole-body composition in a prospective study including six young male SCI patients presenting with an acute complete traumatic paraplegia: bone mineral content (BMC) and density (BMD) of the lumbar spine and of the skull did not vary between the 1st (T1) and the 6th (T6) month after onset. In sharp contrast, in the lower limbs (LL) BMC decreased by $7.1 \%$ and BMD by $6.4 \%$ during the same time whilst there was a $4.8 \%$ increase in the upper limbs (UL) BMC. Whole-body BMC and BMD did not vary significantly between T1 and T6. LL-Lean body mass (LBM) decreased by $10.7 \%$ whereas it increased significantly by $19.6 \%$ in the UL during the same time. The total LBM decreased by $5 \%$ between $\mathrm{T} 1$ and $\mathrm{T} 3$, but no further loss could be measured up to T6. LL-Fat body mass (FBM) increased by $39 \%$ but no change was seen in the UL during the time. The total FBM increased by $23 \%$ between T1 and T6 (D Uebelhart et al, personal results). Bone mass homeostasis is difficult to assess precisely, but most authors agree that major bone loss occurs during the first 6 months after spinal cord injury, and stabilizes between $12-16$ months at $\sim 2 / 3$ of original bone mass, near the fracture threshold. Based upon available data, the overall bone loss occurring during the first months after onset was certainly overestimated by previous studies, mainly because of the large imprecision of the DPA technique used.

Paralysis is accompanied by a broad spectrum of metabolic disturbances which can be evaluated by various biological measurements. In addition to the well known hypercalcemic and hypercalciuric response which is due to the massive mobilisation of calcium from the mineral phase of bone tissue, ${ }^{9-12}$ the organic phase of bone is equally affected by paralysis, as is suggested by the increased initial urinary excretion of hydroxyproline which returns to baseline pre-injury levels between 6-12 months after the onset of the paralysis. ${ }^{13-14}$ Recently, more sensitive and/or specific biochemical markers of bone metabolism have been measured in SCI patients. For instance osteocalcin (OC), a marker of bone formation, was found to be increased in $13 \mathrm{SCI}$ patients in a recent cross-sectional study of Pietschmann et al,${ }^{14}$ whereas in six patients followed longitudinally, normal OC levels were found at one month after injury but $\mathrm{OC}$ values continuously increased thereafter during the 6 months of follow-up. Zanone et al found the same trend in a longitudinal study including $41 \mathrm{SCI}$ patients; OC levels were in the lower range of the normal values by the end of the first month after onset and then increased to peak at month 7 after onset. ${ }^{15}$ Bergmann et al found a similar increase in serum OC in 11 paraplegic patients followed up for 3 months. ${ }^{7}$ The initial depression in the osteoblastic response measured by OC was recently confirmed by our group as well as a decrease in the biosynthesis of type I collagen assessed by the measurement of type I procollagen levels in serum. ${ }^{16}$ It is interesting that in growing rats, Wakley et al showed that periosteum isolated from tibiae and femurs had decreased mRNA levels for osteocalcin, alkaline phosphatase and eventually the prepro-alpha I subunit of type I collagen between 1 and 2 weeks after they were submitted to unilateral sciatic neurectomy. ${ }^{17}$ In SCI patients, Pietschmann et al found increased urinary hydroxyproline/creatinine levels which peaked at month 4 after injury, and increased urinary calcium/creatinine which peaked at month 2. Serum levels of intact parathormone, which represents the biologically active form of the hormone, were low initially and became normal 6 months after the onset. ${ }^{14}$ Bergmann et al measured tartrate-resistant acid phosphatase (TRAP) levels in the serum, a marker of osteoclastic activity. TRAP levels were in the upper range of the controls during the first month after onset and decreased thereafter. ${ }^{7}$ Recently we demonstrated a dramatic and continuous increase in sensitive bone resorption markers such as urinary pyridinoline and deoxy-pyridinoline and serum carboxy-terminal telopeptide of type I collagen beginning as early as 2 weeks after onset of SCI. ${ }^{16}$ Additional data from our group suggest that a peak in bone resorption occurs between 2-3 months after onset (D. Uebelhart et al, personal results). In addition we showed that serum type III procollagen were dramatically increased after SCI, suggesting an increased turnover of connective tissues. ${ }^{16}$

\section{Bone metabolism in simulated weightlessness}

In simulated weightlessness, the effects of immobilisation occur without neurological injury and osteopenia and appear to be primarily due to a reduced rate of bone formation. This was clearly shown in the well described rat model of simulated weightlessness with hind-limbs elevation and unloading while maintaining normal weight bearing on the forelimbs. ${ }^{18}$ Vitamin D metabolism has been shown to be modified by simulated weightlessness in growing animals with a significant decrease in circulating 1,25-(OH)2D after skeletal 
unloading, but it appeared to be secondary to the hypercalcemic response observed in the animals. ${ }^{19}$ In addition, there is recent evidence that skeletal unloading induces a resistance to IGF- 1 action. ${ }^{20}$ Recently, Genty et al used the tail suspension model of MoreyHolton in adult rats and showed a $40 \%$ decrease of osteoid surfaces after 7 days of suspension in the unloaded left proximal tibia which was associated in vitro with a $70 \%$ decrease of alkaline phosphatase activity of isolated osteoblasts. ${ }^{21}$ If these immobilisation studies clearly indicate that in animals, unloading of a skeletal structure invariably leads to bone loss and decreased bone formation in the affected part, Grynpas et al showed that a postcranial 2 week immobilisation of the jaw bone in young Rhesus monkeys significantly changed the density profile of mandibular bone, an element that retained function during the immobilisation period. In fact, a decrease in the quantity of bone in the lower density fractions could be observed at the end of the immobilisation period, which was associated with a decreased bone OC content, both findings being consistent with a decreased bone formation in the jaw. Because the effect occurred rapidly in a non-immobilised and non-weight-bearing bone and persisted for at least one week after remobilisation, the authors suggested a systemic effect of the immobilisation on the skeleton. ${ }^{22}$

\section{Bone metabolism during spaceflight}

Actual spaceflight has also been reported to have tremendous effects on bone metabolism, but most results are difficult to interpret because of great methodological variation. However, generalised bone loss does not appear to be a consequence of microgravity but mainly occurs in localised skeletal areas according to the level of modelling and remodelling and of the support function of each bone in normal gravity. ${ }^{23}$ Decreased OC gene expression has been reported by Backup et al in the bone tissue of young rats after 4 days of space flight. ${ }^{24}$ Schneider et al studied bone mineral density (BMD) before and after space flight of 131 to 312 days duration in nine cosmonauts of the MIR Space Station. Despite an active exercise program during the period in space, a $7.4 \%$ loss in the pelvis $\mathrm{BMD}, 5 \%$ and $9 \%$ respectively in the femoral neck and trochanter, and a $4.2 \%$ increase in the head BMD were found. Whole-body BMD did not change during the follow-up period, whereas a decreased lean mass and an increased fat content in the lower limbs was found. ${ }^{25}$

\section{Bone metabolism after loss of normal biomechanical function}

Loss of biomechanical function invariably results in subsequent bone loss. For instance, in scoliotic patients immobilised by a plaster jacket, Bronner et al showed a significant reduction in bone formation which was quantitatively greater than the increased resorption which occurred after immobilisation. ${ }^{26}$ Immobilisation of the forelimb of dogs in a spica cast for up to 60 weeks resulted in significant spongy and compact bone loss at the level of the third metacarpal. Histomorphometric analysis showed that it was more pronounced in younger animals which presented with $70 \%$ trabecular and $60 \%$ cortical bone loss after 2 months of immobilisation, compared with respectively, $55 \%$ and $50 \%$ in older animals. Interestingly, the animals remobilised after 32 weeks of casting showed a very significant increase in their bone content clearly suggesting a positive effect of remobilisation on recovery of bone loss during the active phase of disuse. ${ }^{27} \mathrm{By}$ immobilising a rat hind limb by knee tenotomy, Thompson et al were able to show a significant increase of osteoclasts as early as $30 \mathrm{~h}$ after tenotomy, an increase in bone resorption surfaces after $72 \mathrm{~h}$ and a decrease in trabecular bone volume after $240 \mathrm{~h}$ in the tenotomised tibiae. Bone resorption could be prevented by indomethacin, but it did not prevent the decrease of bone formation during the 10 days of the experiment. ${ }^{28}$ Adult male monkeys restrained in a semirecumbent position according to Howard et al ${ }^{29}$ for 7 months demonstrated progressive osteopenic changes in their tibial compact bone. Histologically, osteoblastic activity was suppressed and resorption cavities were increased only in some portions of the total tibial section. ${ }^{30}$ Mechanic et al confirmed these results on adult male pigtail monkeys restrained for 7 months with cancellation and osteopenia in tibial cortical bone, a loss of trabecular bone at the endosteal margin, alterations in the recruitment and activation of osteoclasts and a significant increase in non-mineralized collagen content in total bone. ${ }^{31}$

\section{Bone metabolism in long duration bedrest}

Using long duration bedrest studies, Schneider et al showed that healthy adults immobilised in bed present with a negative calcium balance after 2 weeks and after one month of immobilisation $200 \mathrm{mg}$ of $\mathrm{Ca}$ are lost per day. ${ }^{32}$ LeBlanc et al showed that spinal bone mineral content decreased slightly $(0.9 \%)$ but not significantly after 5 weeks of bedrest. ${ }^{33}$ Another study performed on six subjects lying horizontally in bed showed significant loss of BMD at various sites after 17 weeks of immobilisation: total body $(-1.4 \%)$, lumbar spine $(-3.9 \%)$, femoral neck $(-3.6 \%)$, trochanter $(-4.6 \%)$, tibia $(-2.2 \%)$ and calcaneus $(-10.4 \%)$. After 6 months of reambulation, the calcaneus was the only bone presenting with a total BMD recovery. Interestingly, a redistribution of bone mineral was observed during the bedrest period with an increase in skull BMD. The total-body BMD was highly correlated with the change in total-body calcium determined by calcium balance. ${ }^{34}$ Grigoriev et al measured mineral balances, hormone levels and BMD in nine subjects immobilised in bed with 5 degrees head-tilt down for 360 consecutive days and treated with an exercise program used for cosmonauts with or without EHDP: $900 \mathrm{mg}$ daily. The negative calcium balance was reduced by $50 \%$ for the first 120 days, $80 \%$ for the second 
and $69 \%$ during the third 120 day period in the group treated with exercise and bisphosphonate. Exercise alone had no effect until the second 120 day period when the calcium balance improved $52 \%$. BMD measured in the lumbar spine, femoral neck and diaphysis by DPA did not show any significant variation but femoral neck BMD appeared to decrease slightly in the group which received exercise alone. ${ }^{35}$

Van der Wiel et al evaluated bone metabolism in 14 patients who had a lumbar disc protrusion and who were immobilised in bed for 10 days, and found an increased fasting hydroxyproline/creatinine ratio after 4 days of bed rest which peaked after 10 days. Interestingly, these levels did not return to baseline level 6 weeks after remobilisation. Fasting urinary calcium/creatinine ratio followed a similar pattern. Serum calcium and phosphorus increased significantly during immobilisation. Serum 1,25 Vit D3 decreased significantly due to the increased serum calcium and phosphate levels and reached a nadir one week after remobilisation. Total alkaline phosphatase did not vary during the immobilisation period. ${ }^{36}$ Pedersen et al evaluated serum levels of carboxyterminal propeptide of type I collagen (PICP) and osteocalcin as well as urinary deoxy-pyridinoline (D-Pyr) in nine young women before and after 5 days of complete bed rest. Bed rest resulted in a $14 \%$ decrease in serum PICP accompanied by a fade in normal circadian rythm after 5 days of immobilisation. OC and D-Pyr increased during the same period. ${ }^{37}$

\section{Structural changes in collagen as a molecular basis to explain bone loss in disuse}

Recently, some attention has been given to possible structural abnormalities in the collagen of bone in osteoporotic states. Normal adult bone matrix is mainly composed of type I collagen with only small amounts $(<10 \%)$ of minor collagens type III $(5 \%), \mathrm{V}$, and type VI which are nevertheless believed to play an important role in the organisation of type I fibers. ${ }^{38}$ Based on rat collagen turnover analysis, the half-life of type I collagen is 54.7 days and that of type III collagen 32.9 days. No difference was found between skin and bone collagen turnover. ${ }^{39}$ Analysing bone sections from femoral head and neck regions of osteoporotic patients undergoing hip replacement surgery as compared with normal subjects, Bailey et al showed that total collagen content did not vary between the two populations. In sharp contrast, in osteoporotic bone samples, type III collagen content decreased, hydroxylation of lysine residues was dramatically increased, DHLNL content (a reducible cross-link of collagen) decreased dramatically, but no change could be detected in the pyridinoline content (non-reducible cross-link of collagen). In SCI patients we recently found a dramatic increase in type III procollagen in the serum which could reflect the increased connective tissue metabolism. ${ }^{16}$ In addition, the expression of collagen phenotype appeared to be modified since type VI collagen amount tended to decrease. ${ }^{40}$ An altered level of lysyl hydroxylation of the alpha 2(I) chain of type I collagen in osteoporotic bone has also been demonstrated by Batge et al. ${ }^{41}$ Notbohm et al have demonstrated that the thermostability of type I collagen from osteoporotic patients was reduced and was inversely proportional to their degree of lysyl-overhydroxylation, which could increase type I collagen susceptibility to proteolytic degradation. ${ }^{42}$

\section{Conclusions}

It appears that regional or whole-body bone loss is associated with situations such as bedrest, plaster casting, nerve or tendon section, paralysis and actual or simulated spaceflight. Most of the studies dealing with disuse or immobilisation have focused on the weightbearing skeleton; however there is now some evidence that the effects are not only due to a compromised biomechanical function, some of the changes do have a systematic base, such as in those with paralysis. A major difference between these different situations is the intensity and the timing of the bone metabolic responses which is definitively most important in those with paralysis, as compared with those with bedrest, and with simulated or actual spaceflight. As accurate and sensitive non-invasive techniques became available recently, new exciting information has been provided through whole-body or site specific bone mineral density measurements. In sharp contrast with the advancement made with bone densitometry, biochemical modifications induced by disuse are only poorly understood, mainly because of the lack of sensitive and/or specific markers of bone metabolism. Using sensitive biochemical markers of bone metabolism in association with high precision and accurate densitometric techniques in prospective clinical studies should help to identify the factors responsible for the development of secondary osteoporosis and help to define effective therapeutic strategies aimed at reducing the deleterious effects of disuse.

\section{Acknowledgements}

This work was supported by the Swiss National Foundation for Scientific Research (SCORE Grant No 32-35582.92 and Grant No 32-39768.93).

\section{References}

1 Minaire $\mathrm{P}$ et al. Quantitative histological data on disuse osteoporosis: comparison with biological data. Calcif Tissue Res 1974; 17: 57-73.

2 Minaire $\mathrm{P}$ et al. Marrow changes in paraplegic patients. Calcif Tissue Int 1984; 36: 338-340.

3 Biering-Sorensen F, Bohr HH, Schaadt OP. Longitudinal study of bone mineral content in the lumbar spine, the forearm and the lower extremities after spinal cord injury. Europ J Clin Invest 1991; 20: 330-335.

4 Finsen V, Indredavik B, Fougner KJ. Bone mineral and hormone status in paraplegics. Paraplegia 1992; 30: 343-347.

5 Kiratli BJ. Skeletal adaptation to disuse: longitudinal and cross-sectional study of the response of the femur and spine to immobilisation (paralysis). PhD thesis. The University of Wisconsin-Madison, 1989. 
6 Geusens P. Bone loss in the radius, lumbar spine, femoral neck and total body after paraplegia in men. J Bone Min Res 1992; 7: S-326.

7 Bergmann $\mathrm{P}$ et al. La Démineralisation osseuse chez le paraplégique. Réadaptation/Revalidatie 1991; 1-2: 122-127.

8 Garland DE et al. Osteoporosis after spinal cord injury. J Orthop Res 1992; 10: 371-378.

9 Bergmann $\mathrm{P}$ et al. Longitudinal study of calcium and bone metabolism in paraplegic patients. Paraplegia 1977-1978; 15: 147-159.

10 Griffiths $\mathrm{HJ}$, Bushueff B, Zimmerman RE. Investigation of the loss of bone mineral in patients with spinal cord injury. Paraplegia 1976; 14: 207-212.

11 Claus-Walker $\mathrm{J}$ et al. Hypercalcemia and early traumatic quadriplegia. J Chron Dis 1975; 28: 81-90.

12 Naftchi NE et al. Mineral metabolism in spinal cord injury. Arch Phys Med Rehabil 1980; 61: 139-142.

13 Chantraine A. Clinical investigation of bone metabolism in spinal cord lesions. Paraplegia 1971; 8: 253-269.

14 Pietschmann $\mathrm{P}$ et al. Increased serum osteocalcin levels in patients with paraplegia. Paraplegia 1992; 30: 204-209.

15 Zanone X, Castanier M, Chantraine A. Dosage de l'ostéocalcine chez les blessés médullaires: Etude préliminaire. Réadaptation/Revalidatie 1991; 1-2: 89-93.

16 Uebelhart D et al. Early modifications of biochemical markers of bone metabolism in spinal cord injury patients: a preliminary study. Scand. J Rehab Med 1994; 26: 197-202.

17 Wakley GK, Portwood JS, Turner RT. Disuse osteopenia is accompanied by downregulation of gene expression for bone proteins in growing rats. Am J Physiol 1992; 263: E1029-E1034.

18 Globus RK, Bikle DD, Morey-Holton E. The temporal response of bone to unloading. Endocrinology 1986; 118: $733-742$.

19 Halloran BP et al. The role of 1,25-dihydroxyvitamin D in the inhibition of bone formation induced by skeletal unloading. Endocrinology 1986; 118: 948-954.

20 Bikle DD et al. Skeletal unloading induces resistance to IGF-1. J Bone Min Res 1993; 8: S-155.

21 Genty $\mathrm{C}$ et al. Bone formation response to 7-day tail-suspension in rat: in vitro and in vivo studies. J Bone Min Res 1992; 7: S-217.

22 Grynpas MD, Patterson-Allen P, Simmons DJ. The changes in quality of mandibular bone mineral in otherwise totally immobilized Rhesus monkeys. Calcif Tissue Int 1986; 39: 57-62.

23 Vico L, Alexandre C. Microgravity and bone adaptation at the tissue level. J Bone Min Res 1992; 7: S-445-447.

24 Backup $\mathrm{P}$ et al. Spaceflight results in decreased gene expression for osteocalcin in bone and actin in muscle. J Bone Min Res 1992; 7: S-122.
25 Schneider V et al. J Bone Min Res 1992; 7: S-122.

26 Bronner $\mathrm{F}$ et al. Quantitation of calcium metabolism in postmenopausal osteoporosis and in scoliosis. J Clin Invest 1963; 42: 898.

27 Jaworski ZFG, Uhthoff HK. Reversibility of nontraumatic disuse osteoporosis during its active phase. Bone 1986; 7: 431-439.

28 Thompson DD, Rodan GA. Indomethacin inhibition of tenotomy-induced bone resorption in rats. J Bone Min Res 1988; 3: 409-414.

29 Howard WH, Parcher JW, Young DR. Primate restraint system for studies of metabolic responses during recumbency. Lab Animal Science 1971; 21: 112-1172.

30 Young DR et al. Immobilisation-associated osteoporosis in primates. Bone 1986; 7: 109-117.

31 Mechanic GL et al Nonmineralized and mineralized bone collagen in bone of immobilized monkeys. Calcif Tissue Int 1986; 39: 63-68.

32 Schneider VS, McDonald J. Skeletal calcium homeostasis and counter measures to prevent disuse osteoporosis. Calcif Tissue Int 1984; 36: S-151-S-154.

33 LeBlanc A et al. Spinal bone mineral after 5 weeks of bed rest. Calcif Tissue Int 1987; 41: 259-261.

34 LeBlanc $\mathrm{AD}$ et al. Bone mineral loss and recovery after 17 weeks of bed rest. J Bone Min Res 1990; 5: 843-850.

35 Grigoriev AI et al. Effect of exercise and bisphosphonate on mineral balance and bone density during 360 day antiorthostatic hypokinesia. J Bone Min Res 1992; 7: S-449-445.

36 Van der Wiel HE et al. Biochemical parameters of bone turnover during ten days of bed rest and subsequent mobilization. Bone Mineral 1991; 13: 123-129.

37 Pedersen $\mathrm{BJ}$ et al. Bed rest and osteoporosis alters the circadian rythm of type I collagen synthesis. J Bone Min Res 1993; 8: S-324.

38 Keene DR, Sakai LY, Burgeson RE. Human Bone contains type II collagen, type VI collagen and fibrillin. J Histochem Cytochem 1991; 39: 59-69.

39 Rucklidge GJ et al. Turnover rates of different collagen types measured by isotope ratio mass spectrometry. Biochim Biophys Acta 1992; 1156: 57-61.

40 Bailey AJ et al. Biochemical changes in the collagen of human osteoporotic bone matrix. Connective Tissue Res 1993; 29: 119-132.

41 Batge $\mathrm{B}$ et al. Compositional analysis of the collagenous bone matrix: a study on adult normal and oteopenic bone tissue. Eur J Clin Invest 1992; 22: 805-812.

42 Notbohm $\mathrm{H}$ et al. Comparitive study on the thermostability of collagen I of skin and bone: influence of posttranslational hydroxylation of prolyl and lysyl residues. J Prot Chem 1992; 11: $635-643$. 